\title{
Aprendizaje Basado en Juegos Digitales en Niños con TDAH: un Estudio de Caso en la Enseñanza de Estadística para Estudiantes de Cuarto Grado en Colombia \\ Digital Game Based Learning in Children With AdHD: a Case Study in STATISCTICS TEACHING FOR FOURTH GRADE STUDENTS IN COLOMBIA
}

\author{
Julián MORENO ${ }^{1}$ \\ Verónica VALDERRAMA²
}

\begin{abstract}
RESUMEN: La situación actual de innovación tecnológica en educación introduce una nueva visión del juego digital, resaltando su valor pedagógico. Considerando este panorama proponemos el aprendizaje basado en juegos digitales, más específicamente de tipo multi-jugador masivos en línea, como un escenario propicio para lograr que los niños con TDAH (Trastorno por Déficit de Atención y/o Hiperactividad) mejoren su aprendizaje y fortalezcan significativamente sus habilidades sociales, así como la autorregulación de sus emociones. Para ello llevamos a cabo un estudio de caso en el Colegio Santa Bertilla Boscardin de la ciudad de Medellín, Colombia, con estudiantes del grado cuarto de primaria en la asignatura de estadística, los cuales se dividieron en 17 niños como grupo experimental y 40 como control. Los resultados obtenidos mediante una prueba estandarizada demostraron, con una significancia estadística (valor $p<0.005$ ), no solo los estudiantes con TDAH lograron igualar el rendimiento académico de los estudiantes de control, sino que incluso fueron ligeramente superiores.
\end{abstract}

PALABRAS CLAVE: Educación Especial. Aprendizaje basado en juegos digitales. TDAH. Enseñanza de la estadística. Tabulación y graficación de frecuencias.

ABSTRACT: The current situation of technological innovation in education introduces a new insight into digital games, highlighting their pedagogical value. Considering this panorama, we propose digital game based learning, more specifically with massive multiplayer online games, as a favorable setting to enhance performance by children with ADHD (Attention-Deficit Hyperactivity Disorder) in order to improve their learning and strengthen their social skills, as well as provide for self-regulation of emotions. To this end, we carried out a case study in the Santa BertillaBoscardín primary school from Medellin city in Colombia, with fourth grade students in the subject of statistics, of whom 17 children were the experimental group and 40 were the control group. The results obtained using a standard test showed there was statistical significance $(p$-value $<0.005)$; not only did ADHD students manage to reach the same level of academic performance as control students, but theyalsoachieved slightly higher scores.

KEYWORDS: Digital game based learning. Statistics teaching.ADHD. Frequency tabulation and plotting.

\section{INTRODUCCIÓN}

El trastorno por déficit de atención y/o hiperactividad (TDAH) es definido por la Asociación Americana de Psiquiatría (APA por sus siglas en inglés) ${ }^{3}$ como un síndrome neurológico, caracterizado por hiperactividad, impulsividad e inatención, impropias para el grado de desarrollo del individuo. Según esta misma asociación, es uno de los trastornos más frecuentes, afectando cerca de un 5\% de la población infantil de Estados Unidos, así como un 2.5\% de la población adulta. Entre tanto, según cifras suministradas por el Instituto Tomas Pascual de

\footnotetext{
${ }^{1}$ Docente do Departamento de Ciências da Computação e a Decisão da Universidad Nacional de Colombia. Doutorado em Informática na Educação - UFRGS. Medellín, Colombia. jmoreno1@unal.edu.com

${ }^{2}$ Docente do Colegio Santa Bertilla Boscardín. Mestre no Ensino das Ciências Exatas e Naturais - Universidad Nacional de Colombia. Medellín, Colombia. veronicavalderramagomez@gmail.com

${ }^{3}$ http://www.psych.org
} 
España, estos valores rondan entre el 3 y el 7\% de la población infantil en ese país (PEROTE; SERRANO, 2010).

De manera análoga, la Asociación Brasilera de Déficit de Atención (ABDA) ${ }^{4}$, define al TDAH como un trastorno neurobiológico, que aparece en la infancia y que generalmente acompaña al individuo para toda su vida. Según este organismo, se trata del trastorno más común en niños y adolescentes encaminados a servicios especializados, llegando a una cifra cercana del 3 al 5\%, es reconocido oficialmente por varios países, así como por la Organización Mundial de la Salud (WHO por sus siglas en inglés) 5 .

Ahora bien, aunque la mayor parte de los individuos tienen síntomas tanto de déficit de atención como de hiperactividad/impulsividad, en algunos casos predomina uno u otro. El subtipo apropiado debe indicarse en función del patrón sintomático predominante durante los últimos seis meses (APA, 2000). Siendo así, pueden definirse tres subtipos: con predominio en inatención (TDAH-IA), con predominio en hiperactividad/impulsividad (TDAH-HI), y finalmente el combinado (TDAH-C).

Para ser más precisos, la inatención puede expresarse en forma de falta de atención a los detalles, valga la pena la redundancia, en múltiples contextos como el social, escolar o familiar, principalmente en actividades que requieren procesos cognitivos. Con frecuencia, los individuos parecen estar despistados y se distraen con facilidad ante estímulos triviales o irrelevantes, cambiando de manera constante de una actividad a otra. Así mismo, al participar en conversaciones parecen no escuchar y es común que divaguen entre un tema y otro. En trabajos complejos les cuesta organizar las tareas que deben hacer, teniendo particular dificultad en aquellas que supongan un esfuerzo o concentración constante, en algunos casos evidenciando incluso negativismo hacia ellas. También suelen exhibir incapacidad de seguir reglas tanto complejas como simples.

Por su parte, la hiperactividad puede expresarse mediante la dificultad de permanecer sentado o quieto por largos o incluso medianos períodos de tiempo. A los individuos les resulta difícil realizar actividades sosegadas y suelen hablar mucho y de manera descontrolada. También suelen mover continuamente sus manos y pies, así como cambiar de postura. Por otro lado, la impulsividad puede manifestarse como impaciencia para atender conversaciones e incluso por la tendencia a interrumpir a otros. Los individuos suelen ser impacientes y son propensos a dar respuestas precipitadas. Además de la inadecuación social, la impulsividad potencializa el riesgo de accidentes dado que impide la normal inhibición de actividades potencialmente peligrosas.

La incidencia del TDAH es similar en diferentes regiones del mundo, lo cual indica que el trastorno no es secundario a factores racionales o culturales. De hecho, pese a que por largo tiempo su estudio se enfocó principalmente en occidente, estudios realizados en África, Oriente Medio y Asia arrojaron datos de prevalencia muy similares. Lo que es común alrededor del mundo, es que los portadores presentan alteraciones en la región frontal y en sus conexiones con el resto del cerebro. La región frontal orbital es una de las más desarrolladas en

\footnotetext{
${ }^{4}$ http://www.tdah.org.br
}

${ }^{5}$ http://www.who.int 
el ser humano en comparación con otras especies animales y es responsable por el control del comportamiento, la memoria, la organización, la planeación y la capacidad de prestar atención.

Más específicamente, la alteración en esta región cerebral se presenta en el funcionamiento de los neurotransmisores, principalmente la dopamina y la noradrenalina. Si bien el causante de dicha alteración no es único, si existen evidencias respecto a ciertos factores. El principal de ellos es la genética. Según la APA, tres de cada cuatro niños con TDAH tienen un familiar que sufre del síndrome, la ABDA por su parte afirma que la prevalencia del síndrome entre los parientes de los niños afectados es cerca de dos a diez veces mayor que en el resto de la población, lo cual se conoce como recurrencia familiar. Según Cornejo et al. (2004), existe mayor susceptibilidad de padecer el trastorno en familiares de primer orden (5-6 veces más frecuente), concordancia en gemelos monocigóticos (51-86\%) y dicigóticos (29-33\%). Al parecer sin embargo, los genes no son responsables por el trastorno en sí, sino por una predisposición a sufrirlo.

Otras causas estudiadas son el sufrimiento fetal y la ingesta de sustancias durante el embarazo. En el primer caso, aunque las causas no son claras, diversas investigaciones demuestran que las mujeres que tuvieron problemas en el parto desencadenando sufrimiento fetal, tenían más probabilidades de tener hijos con TDAH. En el segundo caso, investigaciones muestran que las madres con problemas de alcoholismo tenían más probabilidades de tener hijos con TDAH. Lo anterior se debe quizá a que la nicotina y el alcohol, cuando se ingieren durante el embarazo, pueden causar alteraciones en diferentes áreas del cerebro del feto, incluyendo la región frontal orbital.

Ahora, respecto a la evaluación diagnóstica del TDAH esta suele ser básicamente clínica, siendo recomendable en la mayoría de los casos una correcta anamnesis y exploración psicopatológica. Eventualmente se requiere además una exploración neuropsicológica e incluso una evaluación psicométrica. En el caso de la recolección de datos sobre la conducta del individuo, es sumamente importante que la misma no solo provenga de él, sino también de los padres y los profesores (PEROTE; SERRANO, 2010). En ambos casos existen cuestionarios y escalas específicas para realizar el diagnóstico. En el caso de los padres, una de las más conocidas es la escala revisada de clasificación para padres de Conners (o CPRS-R por sus siglas en inglés), la cual tiene dos versiones, una larga con ocho sub-escalas, y una corta con cinco. La larga contempla trastornos de conducta, problemas de aprendizaje, ansiedad/timidez, inquietud/ desorganización, síntomas psicosomáticos, síntomas obsesivo-compulsivos, conducta antisocial, e hiperactividad/inmadurez. Por su parte, la corta contempla las tres primeras de la larga más hiperactividad/impulsividad, y ansiedad. Igualmente, en el caso de los profesores, una de las más conocidas es la escala revisada de clasificación para padres de Conners (o CTRS-R por sus siglas en inglés), la cual contempla cuatro sub-escalas: inatención, problemas de conducta, tensión/ansiedad e hiperactividad, y factor de sociabilidad (CONNERS, 1997).

A manera de resumen, en lo Cuadro 1 se muestran los componentes principales de la evaluación diagnóstica del TDAH en el caso de niños (SOUTULLO; DIÉZ, 2007). 


\begin{tabular}{|l|l|}
\hline \multirow{4}{*}{ Imprescindible } & Entrevista clínica con el nińo \\
\cline { 2 - 2 } & Entrevista con los padres, aplicación de cuestionario \\
\cline { 2 - 2 } & Entrevista con los profesores, aplicación de cuestionario \\
\cline { 2 - 2 } & Valorar si se cumple los criterios DSM-IV o ICD-10 \\
\hline \multirow{4}{*}{ Muy recomendable } & Exploración física, incluyendo pruebas de visión y audición \\
\cline { 2 - 2 } & Pruebas de atención \\
\cline { 2 - 2 } & Evaluación de cociente intelectual \\
\hline \multirow{5}{*}{$\begin{array}{l}\text { Necesarias si se sospecha otra } \\
\text { enfermedad }\end{array}$} & Evaluación pedagógica y pruebas de aprendizaje \\
\cline { 2 - 2 } & Estudio neuropsicológico y pruebas de función ejecutiva \\
\cline { 2 - 2 } & Analítica general \\
\hline & Analítica especial \\
\cline { 2 - 2 } & Electro-encefalograma \\
\cline { 2 - 2 } & TAC craneal \\
\cline { 2 - 2 } & Resonancia magnética cerebral \\
\cline { 2 - 2 } & Cariotipo y estudio genético \\
\hline
\end{tabular}

Cuadro 1 - Componentes en la evaluación diagnóstica del TDAH.

Fuente: elaboración propia.

Los criterios DSM-IV e ICD-10 mencionados en la tabla 1 son definidos por la APA (2000) y la WHO (2010) respectivamente, y se resumen de manera consolidada en lo Cuadro 2.

\begin{tabular}{|c|c|c|}
\hline \multirow{9}{*}{ Inatención } & 1 & $\begin{array}{l}\text { Es incapaz para prestar atención a los detalles junto a errores por descuido en las labores escola- } \\
\text { res y en otras actividades }\end{array}$ \\
\hline & 2 & Exhibe dificultad para mantener la atención en tareas o en actividades lúdicas \\
\hline & 3 & Constantemente parece no escuchar cuando se le habla directamente \\
\hline & 4 & $\begin{array}{l}\text { No sigue instrucciones y no finaliza tareas escolares, encargos, u obligaciones. No se debe a } \\
\text { comportamiento negativista o a incapacidad para comprender instrucciones }\end{array}$ \\
\hline & 5 & Demuestra una disminución de la capacidad para organizar tareas y actividades \\
\hline & 6 & $\begin{array}{l}\text { Evita, le disgusta o es renuente en cuanto a dedicarse a tareas que requieren un esfuerzo mental } \\
\text { sostenido }\end{array}$ \\
\hline & 7 & $\begin{array}{l}\text { Extravía objetos necesarios para tareas o actividades (juguetes, ejercicios escolares, lápices, libros } \\
\text { o herramientas) }\end{array}$ \\
\hline & 8 & Fácilmente se distrae ante estímulos externos \\
\hline & 9 & Con frecuencia es olvidadizo en el curso de las actividades diarias \\
\hline \multirow{5}{*}{ Hiperactividad } & 10 & Muestra inquietud con movimientos de manos o pies o removiéndose en su asiento \\
\hline & 11 & $\begin{array}{l}\text { Abandona el asiento en la clase o en otras situaciones en las que se espera que permanezca } \\
\text { sentado }\end{array}$ \\
\hline & 12 & A menudo corretea o trepa en exceso en situaciones inapropiadas \\
\hline & 13 & $\begin{array}{l}\text { Es inadecuadamente ruidoso o tiene dificultades para entretenerse tranquilamente en actividades } \\
\text { lúdicas }\end{array}$ \\
\hline & 14 & $\begin{array}{l}\text { Persistentemente exhibe un patrón de actividad excesiva que no es } \\
\text { modificable sustancialmente por los requerimientos del entorno social }\end{array}$ \\
\hline \multirow{4}{*}{ Impulsividad } & 15 & Con frecuencia hace exclamaciones o responde antes de que se le hagan las preguntas completas \\
\hline & 16 & Es incapaz de guardar turno en las colas o en otras situaciones en grupo \\
\hline & 17 & A menudo interrumpe o se entromete en los asuntos de otros \\
\hline & 18 & Habla en exceso sin contenerse ante las situaciones sociales \\
\hline
\end{tabular}

Cuadro 2 - Criterios diagnósticos del TDAH según DSM-IV e ICD-10.

Fuente: elaboración propia. 


\section{EL TDAH EN EL CONTEXTO ESCOLAR}

Como es de esperarse, y según las manifestaciones del TDAH mencionadas en la sección anterior (ver por ejemplo Cuadro 2), uno de los contextos en los que individuos con este síndrome se ven más afectados es el escolar. En el caso de los niños, Guimarães e Ribeiro (2010) indican que el TDAH ejerce un fuerte impacto negativo en las actividades escolares, ocasionando dificultades en el aprendizaje así como en el relacionamiento con los compańeros de clase y profesores. De hecho, algunos estudios indican que niños con TDAH presentan un riesgo de fracaso escolar de dos a tres veces mayor que niños sin el síndrome y con una inteligencia equivalente (GORDON, 1991).

Y no son pocos los estudios que demuestran o sugieren que el TDAH está intrínsecamente ligado al bajo desempeño escolar. Minde et al. (1971) examinaron el rendimiento académico de 37 niños diagnosticados con hiperactividad y lo compararon en el de igual número de compañeros de clase no hiperactivos. Los resultados demostraron que los primeros tenían una tasa de fallo significativamente mayor en todas las temáticas analizadas, además que eran inequívocamente señalados por sus docentes por tener muchos más problemas de comportamiento en el salón de clase. Similarmente, Cantwell e Satterfield (1978) analizaron 85 niños hiperactivos con edades entre 6 y 11 años y los compararon contra 54 no hiperactivos significativamente similares a los primeros en cuanto a nivel intelectual pero también en cuanto a factores demográficos como edad, sexo y raza. Se calcularon los rendimientos esperados para todos los niños a partir de un análisis de regresión múltiple usando la edad y el IQ. Luego esos valores se compararon contra los rendimientos reales a partir de una prueba estandarizada. Los resultados indicaron que un porcentaje alto de los niños con hiperactividad obtuvieron resultados por debajo de lo esperado en lectura, escritura y aritmética.

Weiss et al. (1979) realizaron un estudio comparativo entre 75 jóvenes hiperactivos comparándolos con 44 de control y encontraron que en promedio los individuos hiperactivos tenían un menor nivel educativo que sus contrapartes. Adicionalmente, encontraron que dentro este grupo algunos continuaban sufriendo del síndrome infantil híper-cinético y tenían un mayor historial de accidentes de autos. Seis ańos después, Weiss et al. (1985) realizaron un estudio similar, pero esta vez con 63 jóvenes hiperactivos y 41 de control y observaron que dentro de los primeros, solamente un 69\% habían concluido sus estudios secundarios frente a un $90 \%$ del segundo caso.

Lambert e Sandoval (1980) realizaron una investigación sistemática sobre discapacidades en el aprendizaje en una población representativa de individuos con hiperactividad así como en un grupo de control. En total se analizaron los datos de 136 individuos en el primer caso y de 152 en el segundo, todos con edades entre 7 y 16 años. Entre sus hallazgos, encontraron que cerca del $53 \%$ de los individuos con hiperactividad demostraban un rendimiento significativamente bajo. August e Garfinkel (1990) examinaron 115 niños diagnosticados con TDAH y los compararon con 50 niños de control. Encontraron que el primer grupo se desempeńó en promedio significativamente peor comparados contra los de control en pruebas sobre memoria secuencial de atención involucrando control de los impulsos y organización. Además $39 \%$ de ellos exhibían incapacidades específicas para la lectura y demostraron un rendimiento inferior en pruebas sobre vocabulario y gramática. 
Mannuzza et al. (1993) observaron que portadores de TDAH tenían en promedio 2.5 años de estudio menos comparados con jóvenes de control de la misma edad. Además, encontraron que 23\% del primer grupo no terminaron sus estudios de secundaria frente a un 2\% del grupo de control. Estos resultados fueron similares a los de un estudio realizado cuatro años después (MANNUZZA et al., 1997). Por su parte, Faraone et al. (1993), en un estudio realizado con un total de 563 niños, encontraron que más del 50\% de los que sufrían TDAH necesitó de aulas particulares y cerca del 30\% fueron reacomodados en grupos especiales o fueron reprobados.

En el ámbito regional, específicamente en Brasil, Rohde et al. (1999) analizaron un total de 1013 estudiantes entre 12 y 14 años de edad y observaron que el 87\% de los portadores de TDAH eran repitentes de al menos un grado escolar, comparado con un $30 \%$ de los no portadores. Así mismo, encontraron que el 48\% del primer grupo habían sido suspendidos al menos una vez y $17 \%$ habían sido incluso expulsados de escuelas anteriores. Esto frente a un $17 \%$ y $2 \%$, respectivamente, del segundo grupo. También en Brasil, Orlandi et al. (2013) compararon el desempeño escolar en tareas metalingüísticas y de lectura de 20 niños entre cuarto a octavo ańo con edades que oscilaban entre 9 y 13 años, 10 de ellos con TDAH y 10 de control. Encontraron que los nińos del primer grupo presentaron un desempeño inferior en las tareas consideradas más complejas, como la manipulación de sílabas y fonemas, así como en la lectura de palabras irregulares, las cuales exigen retención, análisis y recuperación de información. Resultados similares fueron encontrados por Capellini et al. (2007) analizando una población muy parecida, pero para el caso de para nominación rápida de colores, dígitos, letras y objetos.

\section{El JUEGO DigITAL COMO ESTRATEGIA DE ENSEŃANZA PARA NIŃOS CON TDAH}

El escenario escolar de un estudiante diagnosticado con TDAH resultada en ocasiones adverso y complejo ya que puede manifestar rechazo o apatía frente a las actividades educativas, evitación o fracaso en áreas específicas, así como también conducta disruptiva o negativa en algunas clases. Ante la detección de estas dificultades es preciso e importante cuestionar y revisar las prácticas pedagógicas, a fin de generar estrategias que permitan que esta población se motive frente al proceso de enseńanza/aprendizaje. En consonancia con lo anterior Hallowell e Ratey (1999) mencionan que "Los niños con TDAH necesitan sentirse involucrados en las actividades que realizan. De esta manera pueden sentirse motivados, disminuyendo así la probabilidad de que se desconecten".

Pensando en actividades motivantes, ¿qué puede ser mejor para un niño que el juego? Y ¿por qué no involucrar esta actividad en las aulas de clase? En este sentido, Rodulfo (1996) expresa que "no hay ninguna actividad significativa en el desarrollo de la simbolización del niño, ni en la estructuración del niño que no pase por el jugar”. Así mismo Winnicott (1971) argumenta que "lo natural para un niño es el juego y es, por sí mismo, una terapia”. Si bien estos dos autores se refieren al juego en general, sus afirmaciones cobran más fuerza en la era digital que vivimos hoy en día cuando se trata de juegos digitales. De hecho, según Johnson, Adams e Cummins (2012), la inmersión de los juegos digitales como una herramienta eficaz de aprendizaje, ha suscitado el interés y motivación de muchos docentes, pedagogos y profesionales de las distintas áreas, no sólo por su inmenso potencial en el desarrollo de habilidades trasversales como la motricidad fina, la 
comunicación y la colaboración, sino además por el impacto importante que se espera en el corto y mediano plazo en el campo educativo alrededor del mundo.

Desde el punto de vista neurológico, el juego es facilitador de la sinaptogénesis, es decir, facilita el desarrollo de conexiones sinápticas entre las neuronas y la trasmisión de información entre éstas. La formación de sinapsis, a pesar de que se produce a lo largo de toda la vida de una persona, es especialmente importante en las primeras fases del desarrollo madurativo cerebral de un niño, donde gracias a la plasticidad neuronal el efecto sobre los factores de crecimiento neuronal es mayor (TIZON, 2007).

$\mathrm{Y}$ es que así como en la sección anterior se mencionó que hay numerosos estudios que demuestran que el TDAH está intrínsecamente ligado al bajo desempeño escolar, también hay numerosos estudios que demuestran que los juegos, incluyendo los digitales, pueden servir como enfoque para contrarrestar esta problemática. Prins et al. (2011) por ejemplo, examinaron los beneficios de incluir elementos de juego en pruebas estándar de entrenamiento de la memoria. Más específicamente, examinaron si dichos elementos mejoraban la motivación y el rendimiento general de nińos con TDAH. Para ello examinaron 51 niños diagnosticados con el síndrome con edades entre 7 y 12 ańos y los dividieron aleatoriamente en dos grupos: los que utilizaron la prueba estándar y los que utilizaron la versión de juego. Los resultados que obtuvieron demostraron que el segundo grupo exhibía una mayor motivación (dedicaron más tiempo), tuvieron mejor rendimiento (reprodujeron en promedio más secuencias con menos errores), e incluso se desempeńaron mejor en pruebas post- entrenamiento.

En un estudio sobre el efecto motivacional de los juegos de competencia, Geurts, Luman e Van Meel (2008), examinaron 22 niños con TDAH contra 33 niños de control mediante una prueba estandarizada desarrollada a partir de una tarea conocida como de tipo Eriksen Flanker. Concluyeron que los del primer grupo fueron capaces de ejercer el control cognitivo suficiente cuando estaban motivados y que su desempeño aumentó, pese a tener una velocidad promedio de respuesta menor, cuando notaron que estaban compitiendo con sus pares del primer grupo.

En Brasil, Guimarães, Carvalho e Costa (2007) validaron la utilización de juegos digitales educativos en niños con TDAH haciendo uso de dos formatos diferentes: un juego 2D y un juego 3D dentro de un ambiente de realidad virtual. Para ello compararon 19 niños con TDAH contra el mismo número de niños de control. Los resultados encontrados sugirieron que aunque en el primero formato el primer grupo tuvo un desemeńo significativamente más bajo, en el segundo formato no hubo diferencias significativas entre ambos grupos ni en desempeño ni en motivación.

\section{Propuesta de intervención}

Considerando la evidencia, discutida en la sección anterior, a favor de los juegos digitales como herramienta de apoyo al proceso de enseńanza/aprendizaje en niños con TDAH, se planteó como investigación objeto de este trabajo, determinar el impacto de este enfoque sobre un grupo de estudiantes de básica primaria con este síndrome en el área específica de matemáticas. Para dar más claridad al respecto es necesario describir en mayor nivel de detalle los cuatro elementos previamente mencionados: población objetivo, área de conocimiento, enfoque utilizado y metodología de medición. 
La población objetivo consistió en los estudiantes de cuarto grado del Colegio Santa Bertilla Boscardín Boscardín, ubicado en el barrio Robledo de la comuna siete de la ciudad de Medellín. Las edades de tales estudiantes oscilaban entre los 9 y 10 años y sus estratos socio-económico entre los niveles 3 y 4, en una escala de 1 a 6 . En total fueron 57 estudiantes, 17 de los cuales estaban diagnosticados con TDAH incluyendo los tres subtipos, 6 niños y 11 niñas. Este subgrupo se denominó grupo experimental, mientras que los 40 niños restantes, sin TDAH y repartidos en 12 niños y 28 niñas, sirvió como grupo de control.

Ahora bien, respecto al área de conocimiento se decidió utilizar una que históricamente resulta desafiante tanto para docentes como estudiantes: las matemáticas. Y en el caso de los estudiantes lo anterior no solo para aquellos con TDHA sino para todos en general. De hecho en Colombia según ICFES (2010): "Casi la mitad (44\%) de los estudiantes no alcanza los desempeños mínimos establecidos en la evaluación de esta área al momento de culminar la básica primaria”. Más específicamente, dentro del área de matemáticas se abordó, en el sub-área de estadística, el tema de tabulación y graficación estadística de frecuencias.

En cuanto al enfoque utilizado, dentro del amplio espectro de los juegos digitales, se decidió emplear un tipo particular: juego multi-jugador masivo en línea o MMOG por sus siglas en inglés de Massively Multiplayer Online Game. Una de las características principales de esta modalidad es que permite a los jugadores cooperar y/o competir a una gran escala, generalmente del orden de cientos o más, interactuando de manera significativa con el universo en que se desenvuelven así como entre ellos mismos. En particular se hizo uso de la plataforma de uso libre Erudito ${ }^{6}$, la cual se considera un ambiente virtual de aprendizaje con la particularidad que los cursos son visualizados por los estudiantes como un MMOG.

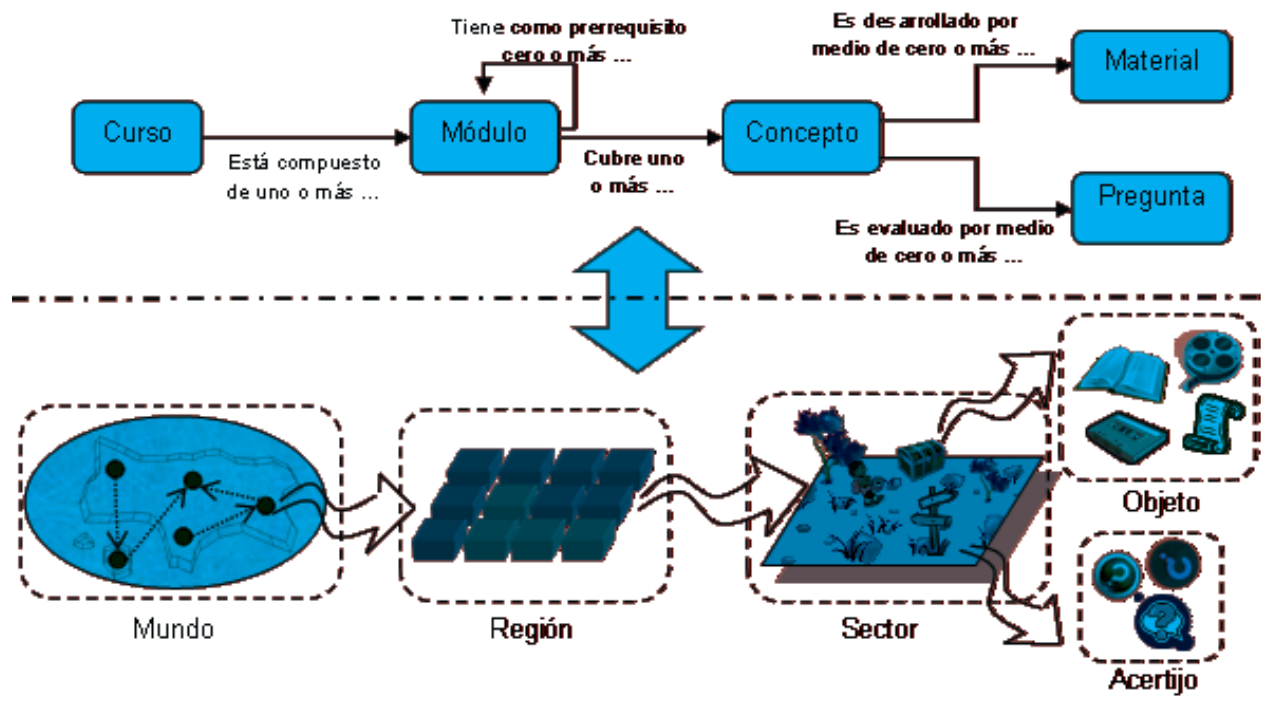

Figura 1 - Metáfora entre curso y juego empleada en Erudito.

Fuente: (MORENO; MONTAÑO; MONTOYA, 2012).

6 http://erudito.medellin.unal.edu.co/ 
Para lograr esto, Erudito emplea una metáfora entre curso y juego como se muestra en la Figura 1, la cual resulta muy natural tanto para profesores como para estudiantes. La temática que se desea abordar se divide en módulos, los cuales abordan conceptos. Para cada concepto existen diversos materiales educativos (videos, textos, imágenes o animaciones), así como preguntas de evaluación. Lo anterior, desde el punto de vista del profesor. Ya desde el punto de vista del estudiante, lo que este experimenta es que hay un mundo, compuesto por regiones, las cuales debe explorar buscando ítems que le ayudan a resolver una serie de acertijos presentados en formato de mini-juegos. Esto, al tiempo que compite con sus compańeros para obtener el mayor puntaje posible.

En la intervención realizada, se creó en Erudito el juego "Palacio de la diosa Safnkit Estocástica” para abordar el tema de tabulación y graficación estadística de frecuencias. Como puede observarse en la Figura 2, dicho juego se dividió en cinco módulos, que en total contenían 35 materiales y 59 preguntas de evaluación. Un ejemplo de la presentación de un material dentro del juego se presenta en la Figura 3.

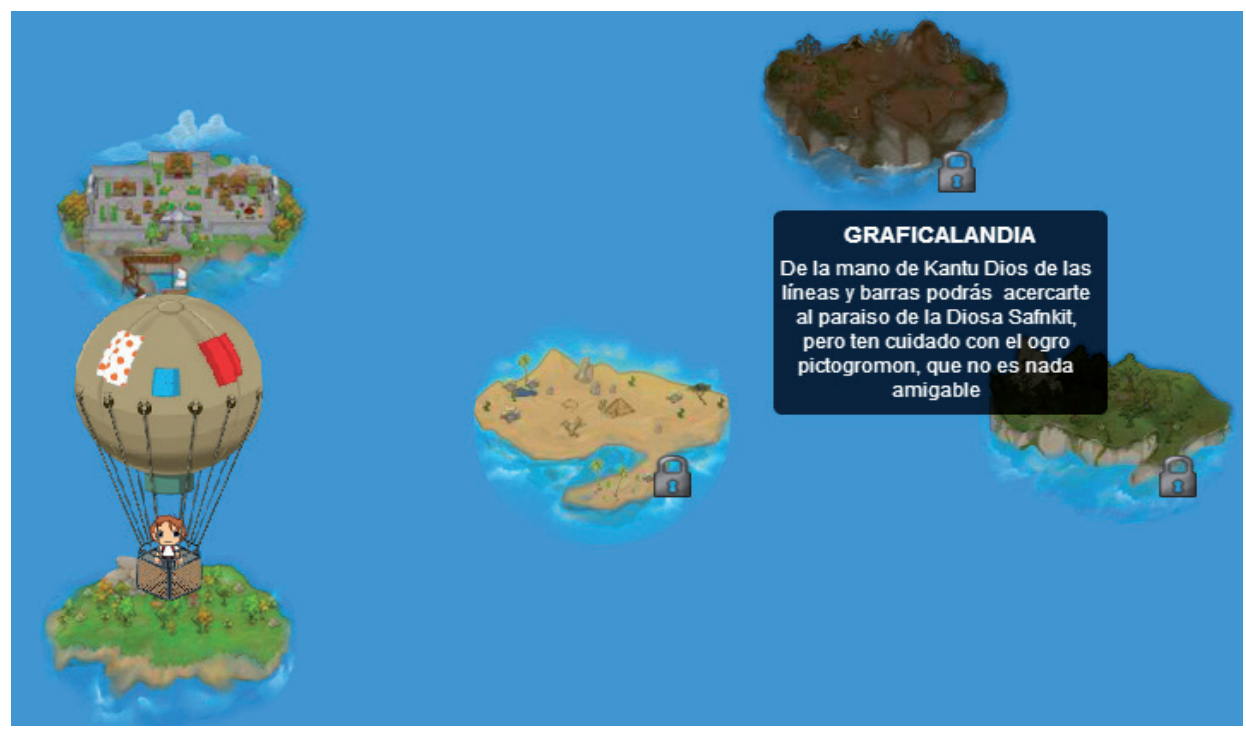

Figura 2 - División del mundo del juego en regiones.

Fuente: elaboración propia. 


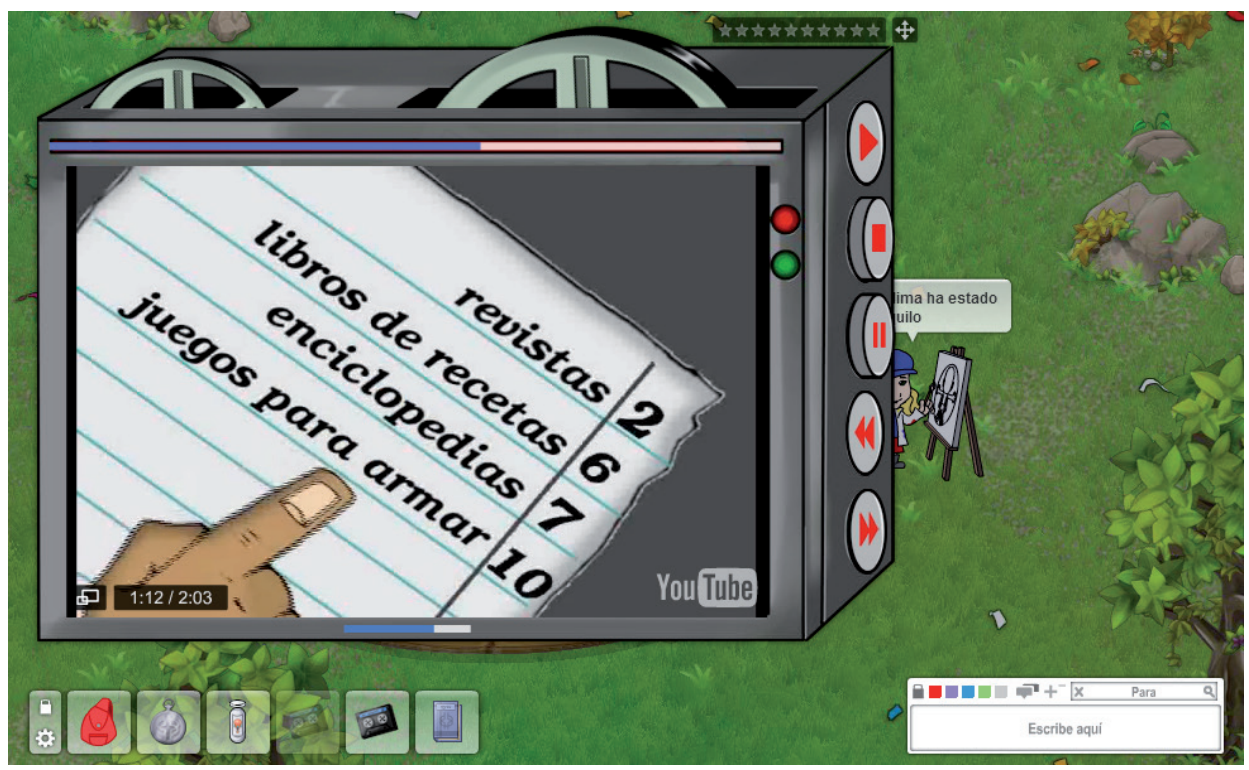

Figura 3 - Presentación de un material educativo.

Fuente: elaboración propia.

Finalmente, en relación a la metodología de medición, se llevó a cabo una comparación cuantitativa de los dos grupos identificados: el experimental y el de control. Este proceso de dividió en varias etapas. En primera instancia se realizó una prueba escrita individual sobre el tema abordado, la cual sirvió como un pre-test para determinar el rendimiento previo promedio de ambos grupos. Dos semanas después se implementó la intervención, la cual consistió en incluir, dentro de las aulas magistrales regulares de ambos grupos, sesiones de interacción dentro del juego. Lo anterior, en un período de 8 semanas donde se tuvieron 8 aulas magistrales, cada una de 45 minutos y 8 sesiones de juego con la misma duración. Cabe anotar que solo se contaba con una sala de sistemas compuesta por 40 computadores, donde sólo 20 de ellos disponían de internet. Por esta razón los estudiantes del grupo experimental pudieron tener las sesiones de a uno por computador, mientras que los de control debieron hacerlo en parejas. Por último, se realizó otra prueba escrita individual, nuevamente sobre el tema abordado, la cual sirvió esta como post-test de rendimiento. Tal prueba fue análoga al pre-test, en el sentido de extensión, dificultad y cubrimiento.

\section{RESULTADOS Y DISCUSIÓN}

El histograma de los resultados del pre-test de rendimiento se presenta en la Figura 4 considerando una escala numérica discreta entre 0 y 5 con una sola cifra decimal. Como puede observarse en dicha figura tales resultados son relativamente uniformes. De hecho, al realizar una prueba de bondad de ajuste aplicando Kolmogorov-Smirnov se obtuvo, con un nivel de significancia del 95\%, que tanto en el grupo de control como en el experimental los datos se distribuyen de forma normal (valores $p$ de 0.078 y 0.060 respectivamente). 


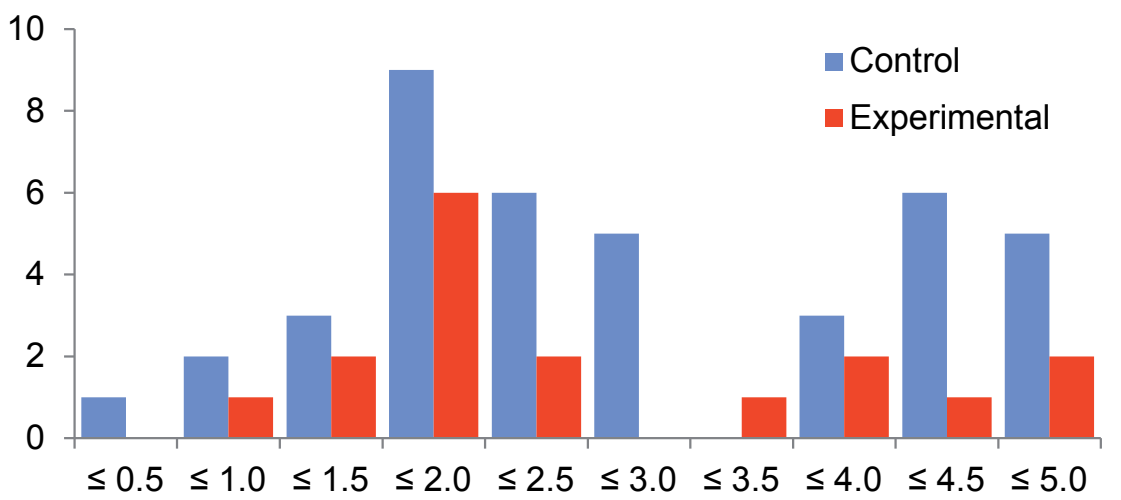

Figura 4 - Histograma de resultados del pre-test de rendimiento.

Fuente: elaboración propia.

Un panorama muy diferente se presenta en el caso de los resultados del post-test. Como se muestra en la Figura 5, en este caso los datos están más inclinados hacia el límite superior de la escala de medición, siendo más evidente en el grupo experimental. En este caso las pruebas de bondad de ajuste arrojan que en ambos casos los datos no se distribuyen de forma normal. Lo anterior según un valor $p$ inferior a 0.01 para el grupo de control y también inferior a 0.01 para el grupo experimental.

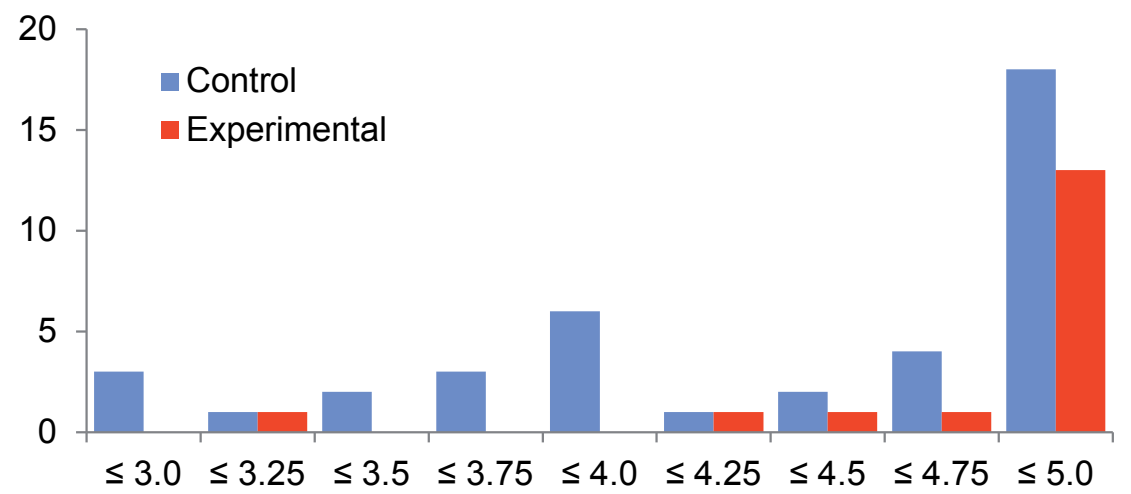

Figura 5 - Histograma de resultados del post-test de rendimiento.

Fuente: elaboración propia.

Ahora, para analizar los datos anteriores con más detalle se realizaron una serie de pruebas de hipótesis para determinar diferencias entre los grupos. Los datos utilizados para dichas pruebas, y que resumen los resultados obtenidos, se presentan en la Tabla 1. 
Tabla 1 - Resumen de resultados de los pre y post test de rendimiento.

\begin{tabular}{l|c|c|c|c|c|c}
\hline \multirow{2}{*}{ Grupo } & \multicolumn{4}{|c|}{ Pre-test } & \multicolumn{3}{c}{ Post-test } \\
\cline { 2 - 7 } & $\mathrm{N}$ & Media & $\begin{array}{c}\text { Desviación } \\
\text { estándar }\end{array}$ & $\mathrm{N}$ & Media & $\begin{array}{c}\text { Desviación } \\
\text { estándar }\end{array}$ \\
\hline Control & 40 & 2.8150 & 1.2967 & 40 & 4.3000 & 0.6854 \\
\hline Experimental & 17 & 2.5647 & 1.2505 & 17 & 4.7118 & 0.4581 \\
\hline
\end{tabular}

Fuente: elaboración propia.

Más específicamente se contrastó la hipótesis nula de que las medias eran iguales contra la hipótesis alterna de que no lo eran. En el caso de los resultados del pre-test se obtuvo un estadístico $t$ de 0.6837 con un correspondiente valor $p$ de 0.4969 . En otras palabras hay evidencia estadística de que tanto el grupo de control como el experimental tenían un rendimiento similar respecto al tema abordado antes de comenzar la intervención. Esto pese a un rendimiento superior cercano al 10\% a favor del grupo de control.

Ahora, al comparar los resultados antes y después de la intervención, es decir, el pre-test contra el post-test, se evidencia una clara mejoría en ambos grupos: cerca del 53\% para el grupo de control, y cerca del $84 \%$ para el grupo experimental. De hecho, los valores $p$ en ambos casos son inferiores a 0.005 con lo cual se rechaza la hipótesis nula en favor de la alterna.

Finalmente, al comparar los resultados de los post-test se obtiene un valor $Z$ de -2.6533 con un correspondiente valor $p$ de 0.006 para un nivel de significancia del $95 \%$. En otras palabras, hay evidencia estadística de que el grupo experimental obtuvo un rendimiento superior al de control. Esto pese a que la diferencia entre ambos al igual que en el pre-test es cercana al $10 \%$.

Todos los hallazgos anteriores se pueden sintetizar en que la intervención realizada, esto es, el juego digital educativo, ayudó a mejorar el rendimiento de ambos grupos, siendo más evidente en el caso del grupo experimental, los niños con TDAH.

\section{Consideraciones finAles}

El TDAH es un síndrome que afecta una proporción considerable de la población en todo el mundo y que le significa a los individuos que lo padecen, principalmente nińos, una serie de dificultades en sus contextos familiar, social y escolar. Particularmente en este último, numerosos estudios han demostrado la alta correlación entre el TDAH y el bajo desempeño escolar, lo cual lo convierte en un asunto de preocupación para docentes, padres de familia, y por supuesto a los niños en sí. Pese a este panorama, existen también investigaciones que demuestran que diversos enfoques, entre ellos el aprendizaje basado en juegos digitales, puede ayudar a atenuar tales efectos negativos. Esto se logra principalmente aumentando la motivación para atenuar la inatención, así como canalizando positivamente la hiperactividad/impulsividad.

En este orden de ideas, el trabajo presentado en este artículo buscaba corroborar el potencial de tal enfoque con una población de niños de cuarto grado de básica primaria diagnosticados con TDAH en el área de matemáticas. Esto no solo se logró, sino que los resultados obtenidos superaron incluso las expectativas más optimistas. Al utilizar una metodología de 
tipo pre-test post-test con un grupo experimental (17 niños con TDAH) y un grupo de control (40 niños sin TDAH) se logró determinar que: a) antes de cualquier intervención ambos grupos mostraban un rendimiento similar, siendo levemente mejor el del grupo de control; b) luego de la intervención los dos grupos evidenciaron una notable mejoría en cuanto a sus rendimientos; y c) dicha mejoría fue ligeramente superior (cerca de 10\%) en el caso del grupo experimental.

Sobre las anteriores conclusiones es importante hacer dos salvedades. La primera es que la mejoría en los rendimientos es atribuible en parte al uso del juego digital considerando que el resto de variables permanecieron iguales para los dos grupos: docente, contenidos y evaluación. Decimos entonces 'en parte' pues dicha intervención se llevó en paralelo con las clases magistrales, siendo difícil, al menos desde los resultados estadísticos, diferenciar los efectos. Lo que si podemos afirmar sin embargo, a partir de las declaraciones de los mismos estudiantes al terminar la intervención, es que el uso del juego digital produjo un enorme efecto en su compromiso hacia el curso. Esto debido principalmente al sentimiento de estar aprendiendo por medio del juego y de estar compitiendo de forma lúdica, sin presiones académicas, con sus demás compañeros.

Lo anterior en el caso de los niños con TDAH es incluso más evidente dado que el juego digital les permite desarrollar sus capacidades en ambientes desafiantes y libres de riesgos, no solo académicos como recién comentamos, sino también físicos. En consonancia con esto, otro hallazgo de interés es que, tal como se mencionó en la introducción, y según lo estipula el manual de trastornos mentales de la APA, una de las características de los individuos con TDAH es su incapacidad para seguir reglas. Sin embargo, el experimento realizado demostró que esto no necesariamente aplica cuando se trata de "reglas de juego". En otras palabras, el problema pareciera no fueran las reglas en sí, más la forma en que se presentan. En el caso de un juego tales reglas parecen ser percibidas menos rígidas y estrictas, por el contrario, más retadoras e incluso divertidas.

Para terminar, la segunda salvedad es que el rendimiento superior en el post-test de los niños con TDAH parece ser contra-intuitivo. Es decir, lo esperado es que se acercara al del grupo de control o que fueran similares con una significancia estadística alta. Nuestra hipótesis frente a tal resultado es que de haber tenido el mismo tiempo de exposición por estudiante en el juego, el desempeño del grupo de control hubiera sido igual de sobresaliente. Vale la pena recordar que por limitaciones de infraestructura, en el caso del grupo de control los estudiantes debieron trabajar de a dos por computador. Sin embargo, independiente de tal fenómeno, lo verdaderamente notable es el efecto catalizador que el juego demostró tener en el aprendizaje de ambos grupos.

\section{REFERENCIAS}

AMERICAN PSYCHIATRIC ASSOCIATION (APA). Diagnostic and Statistical Manual of Mental Disorder. 4th edition. Text Revision (DSM-IV-TR). Washington: American Psychiatric Association, 2000.

AUGUST, G., GARFINKEL, B. Comorbidity of ADHD and reading disability among clinic-referred children. Journal of Abnormal Child Psychology, v.18, n.1, p.29-45, 1990. 
CANTWELL, D.; SATTERFIELD, J. The prevalence of academic underachievement in hyperactive children. Journal of Pediatric Psychology, v.3, n.4, p.168-171, 1978.

CAPELLINI, S. et al. Desempenho de escolares bons leitores, com dislexia e com transtorno do déficit de atenção e hiperatividade em nomeação automática rápida. Revista da Sociedade Brasileira de Fonoaudiologia, São Paulo, v.12, n.2, p.114-119, 2007.

CONNERS, C. Conners' Rating Scales - Revised. North Tonawandda: Multi-Health Systems, 1997.

CORNEJO, W. et al. Caracterización clínica y simulaciones de poder para ligamiento genético en el trastorno por déficit de atención con hiperactividad en familias antioqueńas. Revista de Neurología, v.38, n.4, p.319-322, 2004.

FARAONE, S. et al. Intellectual performance and school failure in children with attention deficit hyperactivity disorder and their siblings. Journal of Abnormal Psychology, Rockville Pike Bethesda, v.102, n.4, p.616-623, 1993.

GEURTS, H., LUMAN, M., VAN MEEL, C. What's in a game: the effect of social motivation on interference control in boys with ADHD and autism spectrum disorders. Journal of child psychology and psychiatry, and allied disciplines, Rockville Pike Bethesda, v.49, n.8, p.848-57, 2008.

GORDON, M. ADHD/Hyperactivity: a consumer's guide. New York: GST Publications, 1991.

GUIMARÁES, M., CARVALHO, L., COSTA, R. Ambientes virtuais na prática educacional de crianças com transtorno de déficit de atenção e/ou hiperatividade. In: SYMPOSIUM ON VIRTUAL AND AUGMENTED REALITY, 9., 2007, Petrópolis. Anais...CERV: Petrópolis, 2007.

GUIMARÁES, M., RIBEIRO, P. Utilização de jogos virtuais na prática educacional de crianças com transtorno de déficit de atenção e/ou hiperatividade. In: SBGames, 9., 2010, Florianópolis. Anais eletrônicos...SBC: Florianópolis, 2010.

HALLOWELL, E., RATEY, J. Driven to distraction (revised): recognizing and coping with attention deficit disorder. New York: Anchor Books, 2011.

INSTITUTO COLOMBIANO PARA EL FOMENTO DE LA EDUCACIÓN SUPERIOR (ICFES). SABER $5^{\circ}$ y $9^{\circ}$ 2009, Resultados nacionales, Resumen ejecutivo. Bogotá: ICFES, 2010.

JOHNSON, L., ADAMS, S., CUMMINS, M. The NMC Horizon Report: 2012 K12 Edition. Austin: The New Media Consortium, 2012.

LAMBERT, N., SANDOVAL, J. The prevalence of learning disabilities in a sample of children considered hyperactive. Journal of Abnormal Child Psychology, v.8, n.1, p.33-50, 1980.

MANNUZZA, S. et al. Adult outcome of hyperactive boys. Educational achievement, occupational rank, and psychiatric status. Archives of General Psychiatry, v.50, n.7, p.565-576, 1993.

MANNUZZA, S. et al. Educational and occupational outcome of hyperactive boys grown up. Journal of the American Academy of Child and Adolescence Psychiatry, v.36, n. 9, p.1222-1227, 1997.

MORENO, J., MONTAÑO, E., MONTOYA, L. Creación y monitoreo de video juegos educativos multi-jugador masivos en línea. In: CONFERENCIA LATINOAMERICANA DE OBJETOS DE APRENDIZAJE - LACLO, 7., Guayaquil. 2012.

MINDE, K. et al. The hyperactive child in elementary school: a 5 year, controlled, follow up. Exceptional Children, Rockville Pike Bethesda, v.38, p.215-221, 1971. 
ORLANDI, V. et al. Desempenho de escolares com transtorno de déficit de atenção e hiperatividade em tarefas metalinguísticas e de leitura. Revista CEFAC, São Paulo, v.15, n.1, p.40-50, 2013.

PEROTE, A., SERRANO, R. TDAH: Origen y desarrollo. Madrid: Instituto Tomas Pascual, 2010.

PRINS, P. et al. Does computerized working memory training with game elements enhance motivation and training efficacy in children with ADHD? Cyberpsychology, Behavior and Social Networking, Rockville Pike Bethesda, v.14, n.3, p.115-122, 2011.

RODULFO, R. El niño y el significante. Buenos Aires: Editorial Paidós, 1996.

ROHDE, L. et al. ADHD in a school sample of brazilian adolescents: a study of prevalence, comorbid conditions and impairments. Journal of the American Academy of Child \& Adolescent Psychiatry, v.38, n.6, p.716-722, 1999.

SOUTULLO, C., DÍEZ, A. Manual de diagnóstico y tratamiento del TDAH. Madrid: Ed. Médica Panamericana, 2007.

TIZON, J. El "niño hiperactivo" como síntoma de una situación profesional y social: ¿mito, realidad, medicalización? Revista de Psicopatología y salud mental del niño y del adolescente, n.2, p.23-30, 2007.

WEISS, G. et al. Psychiatric status of hyperactives as adults: a controlled prospective 15-year follow-up of 63 hyperactive children. Journal of the American Academy of Child Psychiatry, v.24, n.2, p. 211-220, 1985.

WEISS, G. et al. Hyperactives as young adults: a controlled prospective 10-year follow-up of 75 children. Archives of General Psychiatry, v.36, n.6, p.675-81, 1979.

WINNICOTT, D. Realidad y juego. Barcelona: Gedisa, 1971.

WORLD HEALTH ORGANIZATION (WHO). International classification of diseases (ICD). World Health Organization, 2010. Disponível em: <http://www.who.int/classifications/icd>. Acesso em: 01 jul. $2014 .^{7}$

Recebido em: 25/07/2014

Reformulado em: 12/01/2015

Aprovado em: 13/01/2015 
MORENO, J. \& VALDERRAMA, V. 